\title{
Alternatives to standard acute in-patient care for people with mental disorders: from systematic description to evaluative research
}

Michele Tansella

\begin{abstract}
Summary
Many service users and professionals are not satisfied with current hospital care: they call for a safer and more friendly environment, with greater freedom and less social distance between staff and patients. Phase 2 of the Alternatives Study was designed to improve the evidence base for such

residential alternatives. Findings suggest that offering a more acceptable environment increases satisfaction with
\end{abstract}

treatment, although it does not improve the clinical outcome. This set of coordinated studies also suggest that we should listen (and talk) more to our patients, and make our style of working in hospital and community facilities less paternalistic.

\section{Declaration of interest}

None.
The 'balanced model' of the general adult mental health system of care includes a range of service components, such as out-patient or ambulatory clinics, community mental health teams, acute in-patient care, community-based residential care, day care and supported employment. ${ }^{1}$ In-patient care, in modern mental health services, is probably the most problematic component of the system, and many service users and professionals are not satisfied with current hospital care. They call for lower levels of disturbance, a safer and more friendly environment, less coercion, more freedom and less social distance between staff and patients, as well as less paternalistic attitudes by the hospital staff. In particular, women patients sometimes say that they feel vulnerable in such environments. These needs are expressed independently of the severity of the disturbances experienced by service users and of the diagnostic categories to be treated. In many European countries, as well as in the USA, the development of alternatives to hospital has been encouraged in an attempt to meet these needs. The goal has been to organise settings that offer, when an admission is required for the management of acute symptoms, non-clinical environments, less formal staff, shorter admissions and possibly more specific models of care.

In 1971 a model was developed in the USA by Mosher and colleagues to treat people with first-episode schizophrenia outside hospital, in a six-bedroom house on a busy street in a suburban northern California town (the Soteria project). ${ }^{2}$ The intervention was psychosocial with limited antipsychotic medication, and the staff were non-professionals (apart from one psychiatrist) selected on the basis of personal attitudes. The outcome of that experiment was found satisfactory. ${ }^{2}$ The Soteria project was followed by similar experiences in the USA and Switzerland: all these residential alternatives had no more than 15 beds, and were characterised by the home-like, non-institutional atmosphere and by great attention to providing a therapeutic social environment. ${ }^{3}$ The development of these alternatives to acute in-patient care was influenced by the social climate of that period, which was highly critical of all forms of institutional care.

In the past few years, even where community-based mental healthcare is well developed, it remains clear that there is a pressing need to improve hospital acute care, not only in response to demands from service users, their relatives and members of the staff, but also for economic and clinical reasons. In the past 20 years many experiences of residential non-hospital care for acute disorders have accumulated and occasionally been described.
The most comprehensive and accurate description of these alternatives, phase 1 of the Alternatives Study, was recently published by Sonia Johnson et al. ${ }^{4}$ This supplement reports on phase 2 of the Alternatives Study, in which 6 of these 131 alternatives were examined in much greater detail, and also on the Choices Study of women's crisis-house care. ${ }^{5-11}$ These two recent UK studies have aimed to improve substantially the evidence base regarding residential alternatives, allowing clearer conclusions to be drawn about their value as a component within catchment area mental health services. Phase 2 of the Alternatives Study is a multiple methods investigation of six residential and in-patient alternatives to standard acute psychiatric wards in different catchment areas across England: several substudies are described in this supplement. ${ }^{5-10}$ A further paper describes the Choices Study, ${ }^{11}$ a pilot patient-preference randomised controlled trial assessing the effectiveness and cost-effectiveness of two women's crisis houses in two London catchment areas.

\section{Alternatives Study}

The first of the papers on the Alternatives Study showed that in England residential alternatives (clinical crisis houses, crisis team beds, non-clinical alternatives, general therapeutic wards, shortstay wards and general wards for specific groups) are integrated into catchment area mental health systems; they serve similar but not identical patients and provide some but not all of the functions of traditional in-patient care. ${ }^{5}$ This is the first multisite study of this kind and the comparison involved consecutive cohorts of patients admitted to six alternative and six standard acute psychiatric wards. The study concluded that even if these alternative services can divert some patients from acute hospital admission, the resemblances between the two kinds of in-patient care outweigh the differences. Residential alternatives, valued by local stakeholders, are a useful part of the system. I wonder if these alternative services would have an even higher impact on the local system of care, diverting more patients from expensive hospital care, if they could be fully integrated into the system, run under the responsibility of the same consultant who is the clinical lead for the local hospital ward as well as for the other psychiatric facilities in the community. Experiences in some regions of Italy, where the same consultant is responsible for both in-patient and 
community care within a comprehensive and coherent local care system, provide some support for this hypothesis. ${ }^{12}$

A group of 40 purposively selected patients in six residential alternative services, who had previous experience of admission to hospital acute wards, reported an overall preference for the former type of care. ${ }^{8}$ The authors concluded that these alternative services do offer a preferable environment, especially because they minimise coercion and maximise freedom, safety and opportunities for peer support. A further study compared patient satisfaction, ward atmosphere and perceived coercion in community residential alternatives and standard wards, using validated measures: those using the alternative services reported greater levels of satisfaction, having more of a 'voice', greater autonomy, more support, less anger and aggression, and fewer experiences perceived as coercive. ${ }^{9}$

These results could be used for promoting changes in the environment of psychiatric hospital wards, making them more able to 'foster positive interaction while maintaining people's need for privacy and space.8 However, we know how difficult is to change the rules, the norms and the atmosphere in hospitals, which are quite rigid institutions. We might therefore consider transferring the majority of acute psychiatric beds from hospital to mental health centres in the community, for instance, as suggested by the Trieste model. ${ }^{13}$ More research is needed to identify the specific active ingredients of in-patient services that promote improvement. In continental Europe, situating psychiatric wards within general hospital buildings is a general and strongly advocated practice. We consider that admitting psychiatric patients together with all other medical and surgical patients in the same general hospital contributes to decreasing stigma, and encourages collaboration, closer relationships and better links between mental health and other medical and surgical disciplines. This collaboration is useful also for convincing our medical colleagues how important psychological medicine is for improving the diagnosis and outcome of physical illnesses. It promotes the practical implementation of the World Health Organization principle 'no health without mental health'. We should not forget that the inclusion of mental healthcare in the general health system is relatively recent. In Italy, for example, people with mental disorders started to be admitted to general hospitals only in 1978. Before, they were admitted only to remote mental hospitals, or to private psychiatric clinics, away from the rest of medical care. ${ }^{14}$ In the UK, in contrast, locating mental health wards within general hospitals seems to have become substantially less common, and the previous consensus that this is the optimum location seems to have been lost. Little recent literature has explored or evaluated the effects of this important structural factor on patient outcomes and experiences.

\section{Outcomes and cost of alternative services}

The better subjective experience of alternative (compared with standard) services had no clear-cut advantages for short-term outcomes. The improvement was greater for standard services, but only as assessed by Health of the Nation Outcome Scales (HoNOS) measures and in the functioning (but not symptoms) subscale of the Global Assessment of Functioning. However, admissions to alternative services were shorter and significantly cheaper. ${ }^{6}$ Over the medium term, Byford et al explored service use and costs over a 12-month period from date of index admission. ${ }^{7}$ They showed that following discharge from the index admission, mean number and length of readmissions, use of community mental health services and mean cost of further care did not differ between alternative and standard in-patient services.
Shorter length of stay for the index admission in residential alternatives thus explains the lower total 12 -month costs that were found.

The community alternatives were associated with greater service user satisfaction and fewer negative experiences, but not with any better clinical outcomes (improvement in symptoms). These results resemble previous findings of studies comparing assertive community treatment teams and intensive case management with standard psychiatric care. ${ }^{15-18}$ The stakeholders perceived these alternatives as providing more staff time to patients than standard in-patient care, but quantitative assessments found no significant difference in intensity of staff-patient contact. Alternative in-patient services provided slightly more psychological and less physical and pharmacological care than standard wards. ${ }^{10}$ The way in which things are done, rather than what or how much is done, is a key issue in improving patient satisfaction and experience, as well as the quality of the mental healthcare provided and the way it is perceived. This is what has been called 'style of working'. Again, these results call for specific interventions and programmes to improve communication between patients and all healthcare providers, not only mental health staff. Communication skills in the hospital settings can be improved, ${ }^{19}$ and mental health professionals could be on the front line to make these changes in hospital staff.

In this supplement Howard et al report the effectiveness and cost-effectiveness of a specific form of alternative to hospital wards: women's crisis houses. A patient-preference randomised controlled study design was used. ${ }^{11}$ There was no significant difference in outcomes (symptoms, functioning, perceived coercion, stigma, unmet needs or quality of life) or costs between the groups, but women who obtained their preferred intervention were more satisfied. Again, offering a more acceptable environment increases satisfaction with treatment, but does not improve the clinical outcome.

\section{Lessons from research}

The difficulties of achieving randomisation in research studies in acute settings, ${ }^{20}$ and the need to understand the mechanisms influencing outcomes in complex interventions, ${ }^{21}$ including acute in-patient care, support a naturalistic study design using mixed methods and including process research. Such a flexible, mixed approach has been slow to develop in research in acute settings. This may reflect a lack of attention to in-patient care in recent decades, ${ }^{22,23}$ when the research agenda has focused more on assessment of innovations in community-based service provision. This supplement, which includes studies ranging from systematic description to evaluative research regarding alternatives to standard in-patient care, is an excellent example of the usefulness of combining qualitative and quantitative methods in this kind of research. It is an interesting and innovative piece of work that provides information and substantive data on an issue rarely researched before. The main lessons that we learn are twofold: first, we need to provide more personalised care; second, we need to give more attention to patient preferences, choice and control. The aim to increase treatment personalisation is shared also by biologically oriented psychiatrists, who for many years have been looking for laboratory tests, including pharmacogenetic tests, to make possible an individualised, more rational choice of drugs and of drug dosages and regimen. They, as well as our colleagues working in other medical and surgical disciplines, should take advantage also of the second lesson provided by this set of coordinated studies: we need to listen more to our patients, to talk 
more (and better) with them and to make our style of working in hospital and community facilities less paternalistic. We need to take more seriously our patients' need to have a leading role, together with the staff, during the whole process of care.

Michele Tansella, MD, Department of Public Health and Community Medicine, Section of Psychiatry and Clinical Psychology, University of Verona, Policlinico G.B. Rossi, Piazzale L.A. Scuro 10, 37134 Verona, Italy. Email: michele.tansella@univr.it

\section{References}

1 Thornicroft G, Tansella M. Components of a modern mental health service: a pragmatic balance of community and hospital care. Overview of systematic evidence. Br J Psychiatry 2004; 185: 283-90.

2 Mosher LR. Soteria and other alternatives to acute psychiatric hospitalization: a personal and professional review. J Nerv Ment Dis 1999; 187: 142-9.

3 Mosher LR, Burti L. Community Mental Health: A Practical Guide. Norton 1994.

4 Johnson S, Gilburt H, Lloyd-Evans B, Osborn DPJ, Boardman J, Leese M, et al. In-patient and residential alternatives to standard acute psychiatric wards in England. Br J Psychiatry 2009; 194: 456-63.

5 Johnson S, Lloyd-Evans B, Morant N, Gilburt H, Shepherd G, Slade M, et al. Alternatives to standard acute in-patient care in England: roles and populations served. Br J Psychiatry 2010 (suppl 53): s6-13).

6 Slade M, Byford S, Barrett B, Lloyd-Evans B, Gilburt H, Osborn DPJ, et al. Alternatives to standard acute in-patient care in England: short-term clinica outcomes and cost-effectiveness. Br J Psychiatry 2010 (suppl 53): s14-9.

7 Byford S, Sharac J, Lloyd-Evans B, Gilburt H, Osborn DPJ, Leese M, et al. Alternatives to standard acute in-patient care in England: readmissions, service use and cost after discharge. Br J Psychiatry 2010 (suppl 53): s20-5.

8 Gilburt H, Slade M, Rose D, Lloyd-Evans B, Johnson S, Osborn DPJ. Service users' experiences of residential alternatives to standard acute wards: qualitative study of similarities and differences. Br J Psychiatry 2010 (suppl 53): s26-31.

9 Osborn DPJ, Lloyd-Evans B, Johnson S, Gilburt $\mathrm{H}$, Byford $\mathrm{S}$, Leese $\mathrm{M}$, et al. Residential alternatives to acute in-patient care in England: satisfaction, ward atmosphere and service user experiences. Br J Psychiatry 2010 (suppl 53): s41-5.
10 Lloyd-Evans B, Johnson S, Morant N, Gilburt H, Osborn DPJ, Jagielska D, et al. Alternatives to standard acute in-patient care in England: differences in content of care and staff-patient contact. Br J Psychiatry 2010 (suppl 53): s46-51.

11 Howard L, Flach C, Leese M, Byford S, Killaspy H, Cole L, et al. Effectiveness and cost-effectiveness of admissions to women's crisis houses compared with traditional psychiatric wards: pilot patient-preference randomised controlled trial. Br J Psychiatry 2010 (suppl 53): s32-40.

12 Ruggeri $M$, Tansella $M$. Case management or assertive community treatment: are they really alternative approaches? Epidemiol Psichiatr Soc 2008; 17: 93-8.

13 Mezzina R, Vidoni D. Beyond the mental hospital: crisis intervention and continuity of care in Trieste. A four year follow-up study in a community mental health centre. Int J Soc Psychiatry 1995; 41: 1-20.

14 Lasalvia A, Tansella M. Fighting discrimination and stigma against people with mental disorders. Epidemiol Psichiatr SOC 2008; 17: 1-9.

15 Killaspy $\mathrm{H}$, Bebbington $\mathrm{P}$, Blizard R, Johnson S, Nolan F, Pilling S, et al. The REACT study: randomised evaluation of assertive community treatment in north London. BMJ 2006; 332: 815-20.

16 Burns T, Creed F, Fahy T, Thompson S, Tyrer P, White I. Intensive versus standard case management for severe psychotic illness: a randomised trial. UK 700 Group. Lancet 1999; 353: 2185-9.

17 Burns T. Case management or assertive community treatment. What is the difference? Epidemiol Psichiatr Soc 2008; 17: 99-105.

18 Marshall M. What have we learnt from 40 years of research on intensive case management? Epidemiol Psichiatr Soc 2008; 17: 106-9.

19 Smith RC, Dwamena FC, Fortin AH. Teaching personal awareness. J Gen Intern Med 2005; 20: 201-7.

20 Howard LM, Leese M, Byford S, Killaspy H, Cole L, Lawlor C, Johnson S. Methodological challenges in evaluating the effectiveness of women's crisis houses compared with psychiatric wards: findings from a pilot patient preference RCT. J NerV Ment Dis 2009; 197: 772-82.

21 Craig P, Dieppe P, Macintyre S, Mitchie S, Nazareth I, Petticrew M. Developing and evaluating complex interventions: the new Medical Research Council guidance. BMJ 2008; 337: 979-83.

22 Szmukler G, Holloway F. Inpatient treatment. In Textbook of Community Psychiatry (eds G Thornicroft, G Szmukler): 321-33. Oxford University Press, 2001.

23 Bowers L, Chaplin R, Quirk A, Lelliott P. A conceptual model of the aims and functions of acute inpatient psychiatry. J Ment Health 2009; 18: 316-25. 